\title{
Erratum
}

\section{Partial deprivation of GTP initiates meiosis and sporulation in Saccharomyces cerevisiae}

\section{Ashok Varma, Elisabeth B. Freese, and Ernst Freese}

Laboratory of Molecular Biology, National Institute of Neurological and Communicative Disorders and Stroke, National Institutes of Health, Building 36, Room 3D 02, Bethesda, MD 20205, USA

Mol Gen Genet (1985) $201: 1-6$

Please note the following corrections:

Fig. 4 legend: ATP, 0.83 ; GTP, 0.11 ; replace $\mathrm{mmol} / \mathrm{A}_{260} / \mathrm{OD}_{600}$ by nmol/A $\mathrm{A}_{260}$; total Phe-tRNA 2.2 ; charged Phe-tRNA 1.7 ; ratio 0.14 .

Fig. 5 legend: GTP, 0.09 ; replace mmol/ $/ \mathrm{A}_{260} / \mathrm{OD}_{600}$ by nmol/ $\mathrm{A}_{260}$; total Met-tRNA 0.82 ; charged Met-tRNA 0.16.

Fig. 7 legend: GTP, 0.29 ; charged Met-tRNA $0.44 \mathrm{nmol} / \mathrm{A}_{260}$. 